\title{
Distinct Mechanisms Determine $\alpha$-Synuclein Fibril Morphology during Growth and Maturation
}

Arshdeep Sidhu, ${ }^{\dagger, \# \odot ~ I n e ~ S e g e r s-N o l t e n, ~}{ }^{\dagger}$ Vincent Raussens, ${ }^{\ddagger}$ Mireille M. A. E. Claessens, ${ }^{\dagger, \S}$ and Vinod Subramaniam*, ${ }^{*}, \boldsymbol{\&}, \|$

${ }^{\dagger}$ Nanobiophysics, MESA+ Institute for Nanotechnology, University of Twente, Postbox 217, 7500 AE Enschede, The Netherlands

${ }^{\ddagger}$ Structural Biology and Bioinformatics Centre, Structure and Function of Biological Membranes, Faculty of Science, Université Libre de Bruxelles, B-1050 Brussels, Belgium

${ }^{\S}$ MIRA Institute for Biomedical Technology and Technical Medicine, University of Twente, Postbox 217, 7500 AE Enschede, The Netherlands

"Vrije Universiteit Amsterdam, De Boelelaan 1105, 1081 HV Amsterdam, The Netherlands

Supporting Information

ABSTRACT: Amyloid polymorphs have become one of the focal points of molecular studies of neurodegenerative diseases like Parkinson's disease. Due to their distinct biochemical properties and prion-like characteristics, insights into the molecular origin and stability of amyloid polymorphs over time are crucial for understanding the potential role of amyloid polymorphism in these diseases. Here, we systematically study the fibrillization of recombinantly produced human $\alpha$-synuclein $(\alpha$ Syn $)$ over an extended period of time to unravel the origin and temporal evolution of polymorphism. We follow morphological changes in the same fibril sample with atomic force microscopy over a period of 1 year. We show that wildtype (wt) $\alpha$ Syn fibrils undergo a slow maturation over time

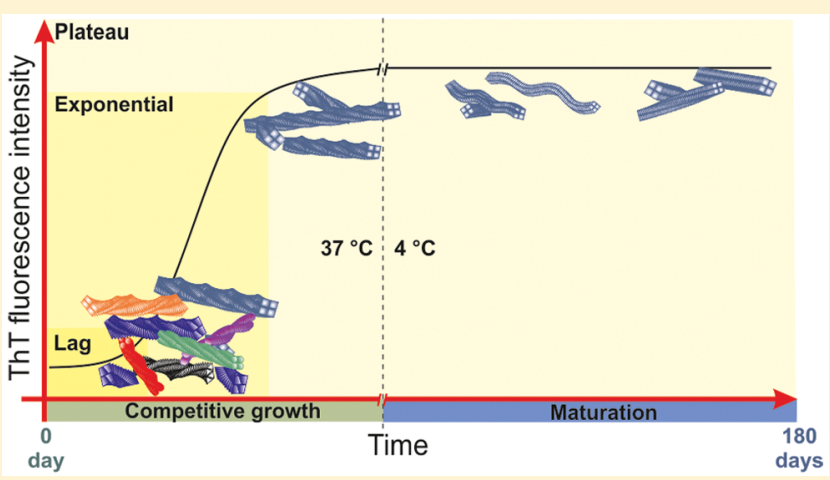
after reaching the plateau phase of aggregation (as detected in a Thioflavin-T fluorescence assay). This maturation, visualized by changes in the fibril periodicity over time, is absent in the disease mutant fibrils. The $\beta$-sheet content of the plateau phase and matured fibrils, obtained using Fourier transform infrared spectroscopy, is however similar for the $\alpha$ Syn protein sequences, suggesting that the morphological changes in wt $\alpha$ Syn fibrils are tertiary or quaternary in origin. Furthermore, results from a reversibility assay show that the plateau phase fibrils do not disassemble over time. Together, the observed changes in the periodicity distributions and stability of the fibrillar core over time point toward two distinct mechanisms that determine the morphology of wt $\alpha$ Syn fibrils: competitive growth between different polymorphs during the fibrillization phase followed by a process wherein fibrils undergo slow maturation or annealing.

KEYWORDS: $\alpha$-Synuclein, amyloid, polymorphism, growth mechanisms, atomic force microscopy

A number of amyloid forming proteins and peptides, including the amyloid $\beta$ peptide $(\mathrm{A} \beta), \alpha$-synuclein $(\alpha$ Syn), and the human islet amyloid polypeptide (IAPP) are implicated in diseases like Alzheimer's disease, Parkinson's disease, and type II diabetes, respectively. ${ }^{1-3}$ Identification of the key factors affecting the conversion of soluble protein molecules into amyloid form and understanding their role(s) in disease is important for designing therapeutics. In vitro, most amyloid forming proteins and peptides spontaneously assemble into fibrils in a wide range of solution conditions. ${ }^{4-12}$ In vitro experiments are thus an important platform to probe the fibrillization process under diverse conditions and to identify mechanisms that may influence the aggregation of the amyloidogenic proteins in vivo.

Fibrillization of soluble amyloidogenic proteins typically follows a sigmoidal growth curve. The plateau phase in
Thioflavin-T (ThT) fluorescence assay is often assumed to indicate the equilibrium in the fibrillization reaction. However, over the past decade, various studies have suggested that the plateau phase is not a true equilibrium in a fibrillization reaction and that slow but significant rearrangement of fibrils takes place over time. ${ }^{12-16}$ Morphological analysis of fibrils by AFM and EM at plateau phase typically shows polymorphic fibrils. ${ }^{6,7,17-20}$ Detailed NMR studies suggest differences in the backbone conformation, electrostatic interactions, and salt bridges in different polymorphs of $\mathrm{A} \beta$ and $\alpha \mathrm{Syn} .{ }^{17,21-24}$ Thus, morphological polymorphism is in all likeliness a consequence of variations in the molecular structure, which in turn arises from

Received: September 2, 2016

Accepted: November 16, 2016

Published: November 16, 2016 

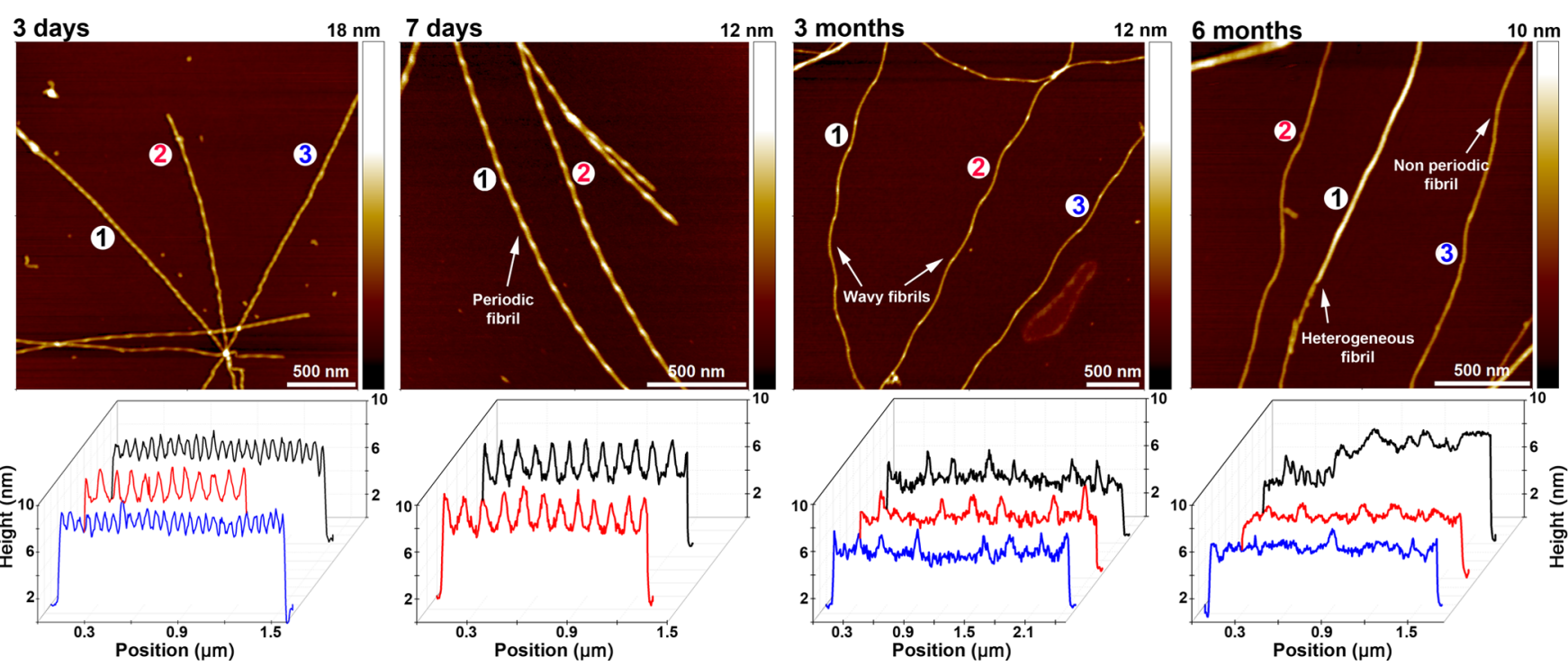

Figure 1. Upper panels, representative AFM height images of wt $\alpha$ Syn fibrils at different time points. Lower panels, color-coded longitudinal cross sections of the numbered fibrils in the upper corresponding AFM images. The cross sections depict the periodicities of the selected fibril. Sample from late lag phase (3 days) shows fibrils with different periodicities. However, fibrils at plateau phase ( 7 days) have similar periodicities. Fibrils at 3 months exhibit larger periodicities with distinct waviness, while samples of 6 months show nonperiodic fibrils and lateral bundling of fibrils termed as heterogeneous fibrils. The height scale in the AFM images is linear.

multiple conformations accessible to the soluble unfolded protein monomer.

The multiple conformations, based on the energy landscape theory for protein folding, could be due to a rough funneled landscape that allows multiple low-energy conformations (local energy minima) in the early stages of folding. ${ }^{25-27}$ The multiple local energy minima are suggested to act as kinetic traps that stabilize partially folded or misfolded conformations. ${ }^{28,29}$ The accessibility of the local energy minima associated with different amyloid conformations are proposed to be critically influenced by the aggregation conditions and the protein sequence. ${ }^{6,9-12,30,31}$ Point mutations in a protein sequence most likely affect the energy landscape resulting in characteristic aggregation behavior. ${ }^{32,33}$ Accordingly, in $\alpha$ Syn aggregations, wt and disease-related mutation sequences are shown to exhibit distinct aggregation kinetics and morphologies. ${ }^{33-42}$

In addition to the proposed differences in the conformation of the proteins leading to distinct fibril morphologies, the polymorphs have been suggested to exhibit different levels of toxicity in cell based studies. ${ }^{21,22}$ Given the observed toxicity profile of distinct amyloid polymorphs, a growing number of studies compare the polymorphic behavior of amyloids to the strain-like properties of prions. ${ }^{21,22,43}$ Like prions, these polymorphs show nucleation (nucleated polymerization behavior), templating (seed aggregations), and cell-to-cell transfer. $^{21,22,43-47}$ Studies on transmission of multiple system atrophy pathology in a transgenic mouse model with complete sets of clinical and biochemical markers provide compelling evidence for the prion-like behavior of $\alpha \mathrm{Syn} .{ }^{48,49}$ However, not much data is available for the competitive growth of different polymorphs in $\alpha$ Syn or other amyloid systems at single fibril level. Competitive growth between prion strains is a hallmark for phenotype determination and is thus one of the critical factors determining the physiological effect in vivo. ${ }^{50-52}$ Longitudinal in vitro studies assessing the relative ratios of the polymorphs over time are valuable to understand the dynamic relation between the experimentally observed morphologies.
In the present study, we systematically investigate and compare the long-term changes in the fibril morphology of wt $\alpha$ Syn and the A30P, E46K, and A53T disease mutants at single fibril level using AFM. The morphology of the helical fibrils was quantitatively studied by measuring their heights and periodicities over a period of 6 months, supplemented with qualitative data at 1 year. Our study provides insight into the changes in the periodicities of the selected $\alpha$ Syn sequences during fibrillization and while in storage. At the applied solution conditions, all the $\alpha$ Syn sequences show polymorphism during the early phase of fibrillization but convert into a fibrillar population of similar morphology on reaching the plateau phase. Moreover, upon long-term storage the periodicities of wt $\alpha$ Syn fibrils progressively transform into a more relaxed twist, while those of disease mutants do not change. Thus, in addition to competitive growth during fibrillization, the wt $\alpha$ Syn fibrils also undergo a process of maturation or annealing.

\section{RESULTS AND DISCUSSION}

Polymorph specific features of amyloids associated with neurodegenerative diseases like Parkinson's ( $\alpha$ Syn) and Alzheimer's disease $(\mathrm{A} \beta)$ are receiving increasing attention due to distinct biophysical, biochemical properties, and prionlike characteristics. Recent studies suggest $\alpha$ Syn to be the first human prion protein. ${ }^{49}$ Despite the emerging body of evidence suggesting $\alpha$ Syn amyloids to be prion-like, little data are available for the growth of different polymorphs with respect to each other. To investigate if the $\alpha$ Syn polymorphs, like prions, grow by competitive growth, we performed aggregation experiments and followed the relative polymorph populations in the same sample over a period of 1 year. Also, we compared the growth of wt $\alpha \mathrm{Syn}$ polymorphs with respect to the three disease associated mutants, A30P, E46K, and A53T, to gain insight on the effect of single amino acid mutation on polymorph specificity.

Fibrillization of Wild-type and Disease Mutants. Wildtype $\alpha$ Syn and the A30P, E46K, and A53T disease mutants were aggregated from monomers in uniform solution 


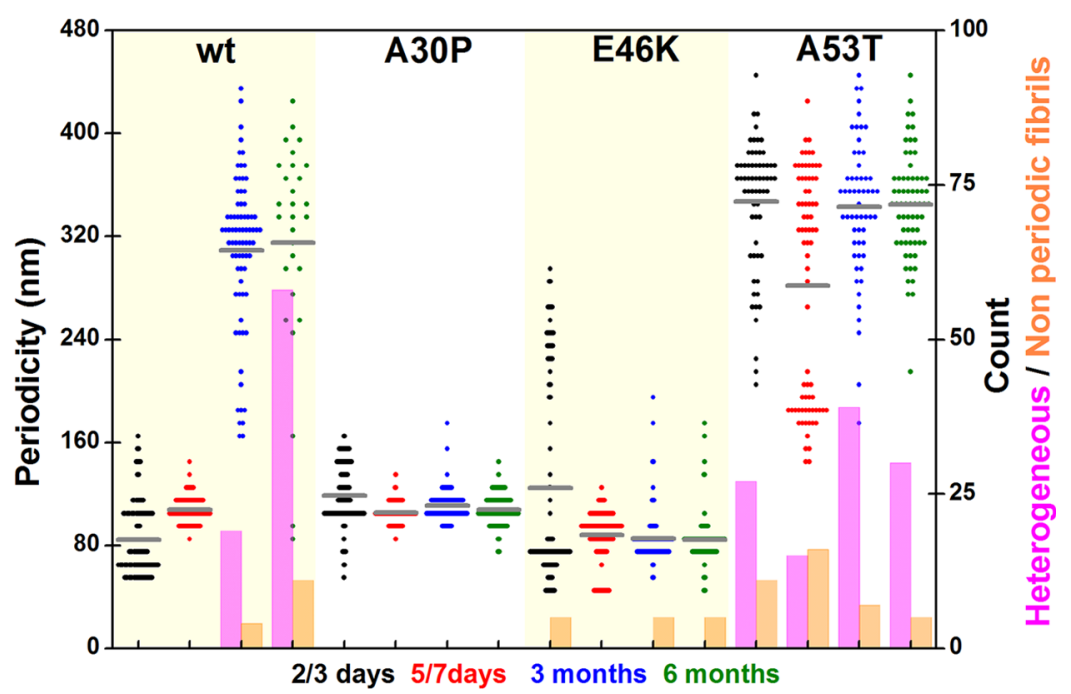

Figure 2. Box plot showing periodicities of wt and disease mutants of $\alpha$ Syn over 6 months. All samples were aggregated in $10 \mathrm{mM}$ Tris-HCl, $10 \mathrm{mM}$ $\mathrm{NaCl}, 0.1 \mathrm{mM}$ EDTA at $\mathrm{pH}$ 7.4. Gray bars represent the mean of the distribution. Pink and orange columns indicate the count of heterogeneous and nonperiodic fibrils. Number of fibrils analyzed, respectively, for each condition, that is, $2 / 3$ days, 5/7 days, 3 months, and 6 months: wt 98 , 105, 99, and 98; A30P 100, 102, 106, and 102; E46K 100, 100, 109, and 103; A53T: 101, 107, 103 and 101.

conditions of $10 \mathrm{mM}$ Tris- $\mathrm{HCl}, 10 \mathrm{mM} \mathrm{NaCl}, 0.1 \mathrm{mM}$ EDTA at $\mathrm{pH} 7.4$ and the fibril formation was followed by a ThT fluorescence assay. The given solution conditions were chosen based on our previous study where inclusion of EDTA resulted in the formation of a homogeneous pool of fibrils at the plateau phase in the ThT assay for wt and A140C mutant $\alpha \mathrm{Syn}^{53}$ In a number of solution conditions the disease mutants of $\alpha$ Syn have been shown to aggregate faster than the wt..$^{34,35,39}$ In our study, A53T fibrillized faster than the other sequences (Supporting Information Figure S1). The lag time was 1-2 days for A53T, A30P, and E46K, while for wt $\alpha$ Syn it was 2-3 days. Correspondingly, the disease mutants reached the plateau phase after 4 (A30P and E46K) or 6 days (A53T), while wt $\alpha$ Syn took around 7 days to reach the plateau.

Fibril Morphology over Time. The aggregations were performed at $37^{\circ} \mathrm{C}$ with shaking and were stored in quiescent conditions at $4{ }^{\circ} \mathrm{C}$ after reaching the plateau phase. The samples were stored at $4{ }^{\circ} \mathrm{C}$ as storage at $37{ }^{\circ} \mathrm{C}$ (without shaking) led to formation of viscous gel-like networks in about $60 \%$ of the samples, most likely due to enhanced interfibrillar interactions. ${ }^{54}$ Highly viscous samples show clustered fibrils even on dilution, which cannot be analyzed at single fibril level by AFM precluding the study of long-term changes. Aliquots of fibril samples were drawn at different time points for AFM imaging. For quantitative morphological analyses of height and periodicity, samples were prepared at 4 time points based on the ThT fluorescence assay: at the end of the lag phase or early fibrillar phase ( 2 and 3 days for mutants and wt $\alpha$ Syn, respectively) to study the morphology of the early fibrillar species, at the plateau phase ( 5 and 7 days for mutants and wt $\alpha$ Syn, respectively), and after 3 months and 6 months to assess the morphology changes of the fibrils during storage. In all the samples, about 100 fibrils were measured at each time point. Figure 1 shows typical morphology of the wt fibrils observed over time.

Early Fibril Samples Show Polymorphism. Samples imaged at 2/3 days by AFM showed long fibrils for wt, A30P, and A53T, while E46K fibrils were shorter and fewer in numbers. Further quantitative analyses of the AFM images revealed a broad distribution of periodicities of the helical fibrils for all samples (Figure 2, Supporting Information Figures S2 and S3 2/3 days, and Table S1). Interestingly in our study, none of the samples showed protofibrils, which according to the proposed hierarchical assembly model $^{7}$ should display fibrils of half heights and periodicities in comparison to mature fibrils. For all the samples, the early fibrillar species showed a distribution of heights and periodicities that were not correlated (Supporting Information Figure S3 2/3 days). We conclude that the fibrils with lower heights are not protofibrils but morphologically distinct mature fibrils. These results clearly show that in the given solution conditions, none of the $\alpha$ Syn sequences studied fibrillize by hierarchical assembly. ${ }^{7}$ Wild-type $\alpha$ Syn fibrils showed two distinct groups of fibrils with periodicities around 50 and $100 \mathrm{~nm}$ and possibly a third fibril species with a periodicity of around $150 \mathrm{~nm}$ (Figure 2 and Supporting Information Figure S3). A30P mutant fibrils did not show any distinct peak in the periodicity distribution but yielded a rather broad distribution from 50 to $150 \mathrm{~nm}$. E46K fibrils had one dominant population with periodicity around 80 $\mathrm{nm}$ but also displayed a broad distribution up to $300 \mathrm{~nm}$ in periodicity. A53T fibrils at 2 days were grouped in three categories: (a) periodic fibrils, which showed a distinct modulation along their lengths with a periodicity of about $350 \mathrm{~nm}$; (b) heterogeneous fibrils, which appeared associated with one or more fibril fragment(s) along their length resulting in an irregular appearance and impeding periodicity analysis; (c) nonperiodic fibrils, which showed no periodicity up to $1 \mu \mathrm{m}$ of length. Another notable feature in A53T aggregations was the presence of wavy fibrils at the end of the lag phase that showed undulation along the fibril length in contrast to the usually straight fibrils (Supporting Information Figure S2). Wild-type and A30P $\alpha$ Syn yielded $100 \%$ periodic fibrils, E46K yielded $94 \%$ periodic fibrils, and A53T had $62 \%$ periodic fibrils in the early fibril samples (Figure 2 2/3 days and Supporting Information Table S2). The mean fibril height in all the samples varied between 5.7 and $6.7 \mathrm{~nm}$ (Figure $32 / 3$ days and Supporting Information Table S3).

Plateau Phase Fibrils Are Homogeneous. On reaching the plateau phase (5/7 days), which is commonly interpreted as the end phase in fibril formation, the morphology of all the 


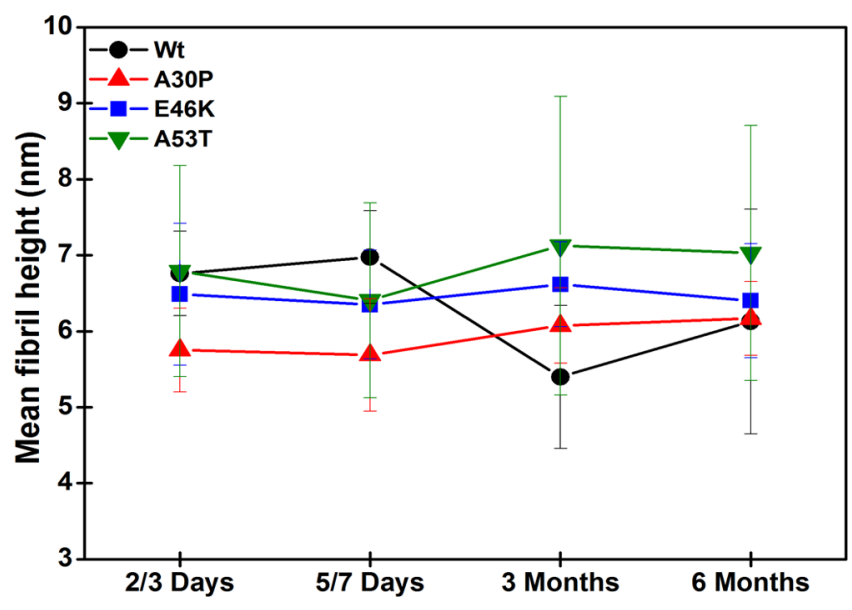

Figure 3. Mean fibril height of wt $\alpha$ Syn and disease mutant fibrils over time. The mean fibril height of the fibrils does not change significantly over a period of 6 months. Error bars denote standard deviation.

samples except A53T became more homogeneous than at the early fibrillar stage (Figure 2, Supporting Information Figures S2 and S3 2/3 and 5/7 days). Although $\alpha$ Syn fibrils are reported to be polymorphic at the plateau phase, ${ }^{8,17,30,55}$ one or more of the polymorphic fibrils could be favored in specific solution conditions to form a near-homogeneous pool of fibrils. $^{22,53}$

Wild-type $\alpha$ Syn at 7 days showed fibrils with average periodicity of $107 \pm 9 \mathrm{~nm}$ in contrast to a periodicity of $84 \pm$ $28 \mathrm{~nm}$ at 3 days. A30P fibrils showed a narrower distribution of periodicities with an average value of $105 \pm 7 \mathrm{~nm}(118 \pm 23$ $\mathrm{nm}$ at 2 days). The periodicity distribution of E46K at $88 \pm 19$ $\mathrm{nm}$ was broader than those of wt and A30P $\alpha$ Syn but was significantly narrower than the periodicity distribution of $\mathrm{E} 46 \mathrm{~K}$ at the earlier time point of 2 days $(124 \pm 80 \mathrm{~nm})$ with a prominent shift toward fibrils of shorter periodicities. A53T fibrils however showed two populations with approximate periodicities of 180 and $350 \mathrm{~nm}$ at 5 days $(282 \pm 87 \mathrm{~nm}$ on average) in comparison to 2 day $(347 \pm 50 \mathrm{~nm})$ fibrils (Figure 2 and Supporting Information Table S1). The heights of the fibrils did not show any major changes and averaged between 5.6 and $6.9 \mathrm{~nm}$ among the fibrils (wt, A30P, E46K, A53T) from the plateau phase (Figure 3 and Supporting Information Table S3).

Our data on the morphology of the fibrils at the late lag phase and plateau phase of ThT assays show that fibril samples that are homogeneous at the plateau phase of the aggregation can show remarkable polymorphism in the lag phase. Polymorphism most likely arises due to accessibility of multiple low-energy conformations in the early stages of aggregation. ${ }^{25-27}$ In intrinsically disordered proteins, like $\alpha \mathrm{Syn}$, the energy difference between the thermodynamically stable conformations (depth of the well) and the kinetic barriers to conversion to another conformation (height of the barrier) are proposed to be very low in the absence of a binding partner. ${ }^{56}$ The conformational dynamics are thus more diverse, and of the numerous conformations in the solution no single conformation is energetically favored. ${ }^{20}$ Moreover, many of the numerous critical nuclei formed during the early events of aggregation can form fibrils of distinct morphology and contribute to the frequently observed polymorphism. ${ }^{17,23,57}$ However, in a particular solution condition one of the polymorphs could be kinetically favored either by higher rate of monomer addition or by pronounced secondary nucleation, that is, fragmentation or surface catalysis. ${ }^{31,50}$ As a result, the selected polymorph incorporates the major fraction of available monomers. Based on our data, the other polymorphs possibly disassemble over time, as fibrils with all but one mean periodicity disappear and monomers reassemble into the dominant morphology observed in the plateau phase fibrils. Therefore, fibrils measured from plateau phase samples in wt and disease mutants, except for A53T, show a narrow fibril periodicity distribution. Notably all four sequences of $\alpha \mathrm{Syn}$ form fibrils of distinct morphology in the same solution conditions (although wt and A30P are very similar). ${ }^{36,40,58-60}$ Given the considerable changes in the distribution of fibril periodicity during the active fibrillization, we decided to follow variations, if any, in the same fibril samples when stored for a long period.

Wild-type Fibrils Mature over Time. The ThT plateau phase fibrils were stored at $4{ }^{\circ} \mathrm{C}$ without shaking, to avoid formation of gel-like networks. At intervals of 2 weeks, 1 month, and 2 months, a qualitative check of the morphology was done by AFM imaging. None of the samples showed major differences in the fibril morphology in comparison to the plateau phase (data not shown). However, at 3 months, the wt $\alpha$ Syn fibrils displayed distinct changes in morphology (Figure 1 ). Only $76 \%$ of the fibrils were periodic with a much wider periodicity distribution and a larger mean periodicity value of $309 \pm 60 \mathrm{~nm}$ (Supporting Information Tables S1 and S2). Twenty percent of the fibrils were designated as heterogeneous and $4 \%$ as nonperiodic. Interestingly, most of these wt fibrils also manifested the wavy feature observed for A53T fibrils at 2 days. Thus, there were striking similarities between wt fibrils of 3 months and A53T fibrils of 2 and 7 days (Figures 1 and 2, Supporting Information Figures S2 and S3, Tables S1 and S2). Fibrils prepared from the A30P, E46K, and A53T variants however did not show any significant change in the mean periodicity of the fibrils over time (Supporting Information Table S1). The E46K sample contained 95\% periodic fibrils, while the A53T sample had $\sim 55 \%$ periodic fibrils (Supporting Information Table S2). The height of wt fibrils was on average $1.5 \mathrm{~nm}$ lower at 3 months than at the plateau phase. This is most likely due to the difference in the ratio of pixels representing the peak and trough heights in AFM images of fibrils of different periodicities (for details refer to Supporting Information Figure S4). The mean fibril height of A30P and E46K fibrils did not change significantly in comparison to fibrils of plateau phase. However, the height of A53T fibrils showed a broader distribution (Figure 3 and Supporting Information Table S3).

Next, the fibrils were analyzed at 6 months following the same procedure. The wt fibrils showed a clear quantitative change in morphology with only $29 \%$ of the fibrils displaying a periodic twist with mean fibril periodicity of $315 \pm 83 \mathrm{~nm}$ (Figure 2, Supporting Information Figure S3, Table S1 and S2). None of the disease mutants appeared to change in periodicities at 6 months. The heights of the wt and A53T fibrils exhibited a broad distribution with a small population showing approximately double heights (Figure 3, Supporting Information Figure S3 and Table S3). This increased height is speculated to be due to bundling or juxtapositioning of the fibrils. A qualitative check at 12 months did not show notable difference in the $\alpha$ Syn fibrils (Supporting Information Figure S5). 
The fibrils at the plateau phase in ThT assays are normally considered to be in equilibrium with the monomers and the intermediate aggregating species and are therefore assumed not to undergo major transitions. However, quantitative measurements on single fibrils by AFM in samples up to 6 months evidently show that wt $\alpha$ Syn fibrils change while they are stored at $4{ }^{\circ} \mathrm{C}$. This is in agreement with recent studies showing that the plateau phase fibrils are in a quasi-equilibrium state. ${ }^{13-16}$ In vivo studies on plaques of $\mathrm{A} \beta$ in transgenic mice, using luminescent conjugated oligothiophene probes, have previously suggested conformational changes in $\mathrm{A} \beta$ fibrils with aging. From in vitro experiments these changes were proposed to arise due to maturation which is postulated to be an intrinsic property of $\mathrm{A} \beta$ fibrils and not because of any extrinsic influence. ${ }^{14}$ It is therefore plausible that wt $\alpha$ Syn fibrils also undergo a maturation process as seen in the present in vitro study. The disease mutants, however, do not show such maturation. In view of the NMR evidence for perturbed structured regions in the mutants in comparison to wt ${ }^{61}$ it is likely that the mutants have distinct preferred conformations, which lead to thermodynamically more stable fibrils. ${ }^{62}$

Fibril Samples Do Not Degrade on Storage. Wild-type $\alpha$ Syn fibrils thus exhibit a maturation reflected in changes in periodicity over time, while the fibrils of the disease mutants do not. To ascertain that this morphological change is not due to degradation of $\alpha$ Syn molecules in the fibrils while in storage, we performed two experiments. First, a dot blot was done on fibrils with antibody against $\alpha$ Syn $15-123$ residues. The antibody was reacted with wt, A30P, E46K, A53T, $\Delta 1-59$ (N-terminal truncation), and $\Delta 109-140$ (C-terminal truncation) $\alpha$ Syn in monomeric and fibrillar (plateau and matured, 1 year old) form. The truncation mutants were used as controls to test the immunoreactivity of the antibody in protein sequences that lack the $\mathrm{N}$ or $\mathrm{C}$ terminus; hence only plateau phase fibrils of the truncation mutants were tested. The antibody reacted well with the monomers of all the sequences showing that even in sequences with $\mathrm{N}$ or $\mathrm{C}$ terminal truncations sufficient binding epitope is available. In fibrillar form, however, plateau and matured fibrils of wt, A30P, E46K, and A53T sequences showed a strong signal while fibrils prepared from $\mathrm{N}$ and $\mathrm{C}$ terminal truncation mutants did not show any reactivity, suggesting that the presence of intact $\mathrm{N}$ and $\mathrm{C}$ termini is essential for the antibody to bind to fibrils (Supporting Information Figure S6). In fibrillar form, the full length fibrils are suggested to have a different structure and morphology than the truncation mutant fibrils, in which the fibril core containing the NAC region of the protein most likely is less accessible for the antibody, resulting in loss of epitope. ${ }^{16,17,22,24,61,63,64}$ Therefore, identical reactivity of anti- $\alpha$ Syn antibody to plateau phase and matured fibrils of wt and disease mutants indicated that the protein molecules in the fibrils had intact epitopes.

Second, we performed MALDI-TOF mass spectrometry on trypsin digested plateau phase and matured fibrils to investigate at residue level if the $\mathrm{C}$-terminus is intact in the fibrils. Trypsin digestion of intact $\alpha$ Syn is expected to yield a number of peptide fragments (Supporting Information Table S4). While an intact $\mathrm{N}$-terminus is difficult to probe with trypsin digestion due to multiple digestion sites within the first 60 residues, an intact C-terminus should generate peptides corresponding to the last 38, 43, and 44 residues (Supporting Information Table S5). In both plateau phase and matured samples, distinct peaks corresponding to an average molecular mass of 4831.02 and 4959.19 Da for $\mathrm{M}+\mathrm{H}^{+}$were observed (Supporting
Information Figure S7). These masses correspond to the 98140 and $97-140$ residues of the C-terminal peptides, respectively (Supporting Information Tables S6 and S7). In addition to hydrogen ion adducts, sodium ion adducts of the peptides were also observed despite changing the buffer to $\left(\mathrm{NH}_{4}\right)_{2} \mathrm{CO}_{3}$. Intriguingly, the matured samples, with the exception of E46K fibrils, showed higher intensity of the sodium ion adducts in comparison to the hydrogen ion adducts. It is likely that the negatively charged fibrils of $\alpha \mathrm{Syn}$ bind positive ions over time, which is reflected in the higher intensity of the sodium ion adducts in matured samples. Additionally, none of the peptides of interest showed $\mathrm{m} / \mathrm{z}$ peaks corresponding to oxidation products of methionines at positions 116 and 127. In a control experiment, we performed the same experiment of trypsin digestion and mass spectrometry on $\alpha$ Syn fibrils prepared from the $\Delta 109-140$ mutant. As expected in the absence of C-terminus no peaks corresponding to the average molecular mass of 4831.02 and 4959.19 Da were observed thus proving that the above-mentioned peaks arise from the designated residues (Supporting Information Figure S8). Therefore, based on the dot blot and mass spectrometry data, we conclude that the protein monomers in the fibrils do not degrade or undergo oxidation while in storage at $4{ }^{\circ} \mathrm{C}$ for at least up to a year.

Plateau and Matured Fibrils Have Similar $\beta$-Sheet Content. To understand further the nature of the morphological differences in wt $\alpha$ Syn in terms of secondary structure, we performed attenuated total reflectance-Fourier transform infrared spectroscopy (ATR-FTIR) experiments on fibrils of wt and disease mutants at plateau phase and 1 year. Deconvolution of the ATR-FTIR spectra followed by a curve-fitting of the amide I peaks showed $\beta$-sheet content of about $65-70 \%$ for all tested fibrils with a major peak located between 1627 and 1630 $\mathrm{cm}^{-1}$ (Table 1 and Supporting Information Figure S9). The

Table 1. Summary of the $\beta$-Sheet Content (\%) of Plateau Phase and 1 Year Old (Matured) Fibrils as Analyzed by ATR-FTIR

\begin{tabular}{ccccc} 
& $\begin{array}{c}\text { wt (max, } \\
\left.\mathrm{cm}^{-1}\right)\end{array}$ & $\begin{array}{c}\mathrm{A30P}(\max , \\
\left.\mathrm{cm}^{-1}\right)\end{array}$ & $\begin{array}{c}\mathrm{E} 46 \mathrm{~K}(\max , \\
\left.\mathrm{cm}^{-1}\right)\end{array}$ & $\begin{array}{c}\mathrm{A53T}(\max , \\
\left.\mathrm{cm}^{-1}\right)\end{array}$ \\
$\begin{array}{c}\text { plateau } \\
\text { phase }\end{array}$ & $68(1627)$ & $66(1629)$ & $66(1628)$ & $68(1629)$ \\
$\begin{array}{c}\text { matured } \\
\text { fibrils }\end{array}$ & $69(1630)$ & $66(1629)$ & $64(1628)$ & $70(1630)$ \\
\hline
\end{tabular}

ATR-FTIR results showed no significant differences in the spectra of plateau phase and matured wt $\alpha$ Syn fibrils or the disease mutants, indicating similar $\beta$-sheet content even in fibrils with distinct morphology. The origin of the observed morphological changes in the fibrils is therefore likely due to tertiary or quaternary structural rearrangements in the fibrils with time.

Two distinct molecular mechanisms could account for the observed morphological changes in wt $\alpha$ Syn. In a competitive growth model, as reported for prion strains, the soluble monomers could adopt different conformations that result in fibrils of different morphologies. These conformations likely compete for the monomer fraction during the aggregation process, and the kinetically favored conformation (faster monomer addition or more breakage) outcompetes the rest at plateau phase. However, once in storage at $4{ }^{\circ} \mathrm{C}$, a different fibril conformation is kinetically and thermodynamically favorable, which accumulates over time. For this mechanism, 
it is imperative that fibril assembly be a reversible process and the fibrils formed at plateau phase fall apart and are recycled into fibrils of a different morphology at $4{ }^{\circ} \mathrm{C}$. In a maturation process, the fibrils formed at plateau phase do not fall apart in storage but undergo a slow transition at a single fibril level and mature over time leading to a thermodynamically more stable conformation.

The first mechanism is evident during the early fibrillization since at early fibrillar phase, fibrils of different morphologies are apparent and one of these morphologies becomes the major population at the plateau phase. However, it is difficult to establish the basis for the long-term transitions occurring at 4 ${ }^{\circ} \mathrm{C}$ in wt $\alpha$ Syn. Assuming there is competitive growth at $4{ }^{\circ} \mathrm{C}$, there are two possible scenarios. In the first instance, we assume that the critical monomer concentration (CMC) required for the formation of aggregating nucleus of $4{ }^{\circ} \mathrm{C}$ conformation is higher than the residual monomer concentration (RMC) at plateau phase (at $37^{\circ} \mathrm{C}$ ). In this scenario, the existing fibrils should cold denature appreciably to provide sufficient monomers to form new fibrils. 22,65

In the second scenario, we assume the $\mathrm{CMC}$ at $4{ }^{\circ} \mathrm{C}$ to be much lower than the RMC at plateau phase. In this case, the amount of monomers in the supernatant at plateau phase is enough for nucleation and fibril growth from the slow dissociation of plateau phase fibrils at $4{ }^{\circ} \mathrm{C}$. If the second scenario holds, an aggregation at $4{ }^{\circ} \mathrm{C}$ with $100 \mu \mathrm{M}$ wt $\alpha$ Syn should form fibrils with morphologies characteristic of stored fibrils.

Plateau Phase Fibrils Do Not Disassemble on Storage. Some recent reports on $\alpha$ Syn have shown that fibrils prepared at $37{ }^{\circ} \mathrm{C}$ can disassemble into monomers within hours when kept at lower temperatures. ${ }^{22,65}$ This reversible nature of the fibrils was however shown to be dependent on the solution conditions used for fibrillization. ${ }^{22}$ We tested for this possibility systematically. Aggregation reactions (wt and mutants) at plateau phase were centrifuged, and the RMC was determined; then new buffer was added to the fibril pellet to restore the original volume, and the fibrils were incubated for a week at 37 ${ }^{\circ} \mathrm{C}$ without shaking to prevent fibril breakage. This procedure of centrifugation and RMC determination was done over 3 weeks collecting three time points. Negligible amounts of monomers were recovered in the second and subsequent RMC analyses. Next, the same procedure was repeated, but the fibrils were stored at $4{ }^{\circ} \mathrm{C}$; this too did not show significant monomer recovery in the supernatant fractions. In addition, ThT analyses from the same samples showed consistent presence of ThT positive $\beta$-sheet structures (Figure 4 and Supporting Information Table S8). Thus, the fibrils do not dissociate at lower temperature of $4{ }^{\circ} \mathrm{C}$, and the overall equilibrium is not shifted toward disassembly under the conditions used in our experiments.

Fibrillization at Low Temperature Produces Periodic Fibrils. To examine if the wt fibril morphology at $4{ }^{\circ} \mathrm{C}$ (i.e., periodicity of about $300 \mathrm{~nm}$ with heterogeneous and nonperiodic fibrils) is a result of a kinetically preferred conformation at low temperature, we aggregated wt $\alpha \mathrm{Syn}$ at $4{ }^{\circ} \mathrm{C}$. Aliquots were withdrawn at an interval of 2 weeks for ThT assay and AFM samples. By the end of 1 month, a few short fibrils could be seen in AFM images but no increase in ThT fluorescence intensity was detected (data not shown). After 3 months, long fibrils similar to fibrils produced at $37^{\circ} \mathrm{C}$ with a periodicity of about $100 \mathrm{~nm}$ were observed. Thus, even at low temperature periodic fibrils appear first. The same fibril

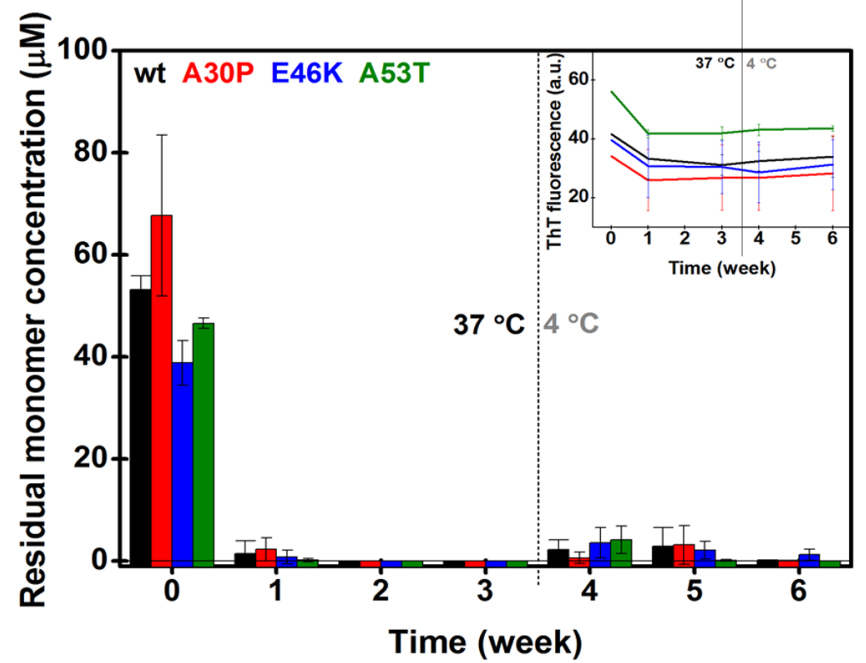

Figure 4. Plot showing irreversible nature of wt and mutant $\alpha$ Syn fibrils prepared in $10 \mathrm{mM}$ Tris- $\mathrm{HCl}, 10 \mathrm{mM} \mathrm{NaCl}, 0.1 \mathrm{mM}$ EDTA at $\mathrm{pH}$ 7.4. Residual monomer concentration determination shows that after removal of monomers from a plateau phase reaction (ThT assay) at week zero, the monomer fraction in the supernatant is not replenished in fibrils stored at 37 or $4{ }^{\circ} \mathrm{C}$. In the inset, a parallel ThT assay every alternate week showed the presence of $\beta$-sheet positive fibrils in reactions over the same period. Error bars denote standard deviation among triplicates.

sample imaged after 1 year showed heterogeneous and nonperiodic fibrils (Supporting Information Figure S10). This suggests that the transitions observed in the fibrillar morphologies are independent of temperature and most likely an inherent characteristic of the wt $\alpha$ Syn fibrils. Moreover, these results show that temperature dependent adaptation of amyloid fibril morphology is not a part of the maturation process observed in our study.

Thus, the morphology of the wt fibrils is governed by two mechanisms. In the aggregation reaction, initially a prion strainlike competitive growth determines the morphology of the plateau phase fibrils. Thereafter a slow maturation process takes over, possibly involving rearrangements in the tertiary or quaternary structure of the fibrils. To comment on the precise nature of the rearrangements between the plateau phase fibrils and the mature fibrils, additional in-depth structural study is required. Further, it is reasonable to assume that the morphological transitions seen over months in wt $\alpha$ Syn could happen at a shorter time scale at $37{ }^{\circ} \mathrm{C}$. We also note that the results presented here were obtained at low salt conditions, and it is difficult to predict whether similar behavior can be expected from $\alpha$ Syn fibrils in vivo, where the solution and molecular milieu is very different. Nonetheless, the present study illustrates the importance of mechanisms such as competitive growth in determining morphological characteristics of fibrils assembled from different $\alpha$ Syn sequences and shows that while wt $\alpha$ Syn exhibits the process of maturation, the disease mutants form morphologically different and more stable fibrils at the solution conditions used.

\section{CONCLUSIONS}

Together our results show that during the early stages of fibrillization several distinct morphologies for all the $\alpha$ Syn sequences exist, providing empirical evidence that the same sequence of the protein can attain a number of conformations 
that form fibrils of different morphologies. This ability to sample various amyloid conformations appears to be an intrinsic property of the polypeptide sequence and bears a resemblance to the strain-like behavior of prions. In complement to the recent reports on prion-like hypotheses for amyloids, based on cell-to-cell transmission of pathogene$\operatorname{sis}^{44,46,48,66}$ our study highlights the property of competitive growth between polymorphs of the misfolded $\alpha$ Syn. Further observation of evolution of wt fibril morphology over time in contrast to the stability of the mutant fibrils' morphology underscores fundamental differences in the way protein molecules with one amino acid difference assemble into fibrils in the same solution conditions. Since the fibrils do not significantly disassemble or degrade when stored, it is likely that the wt fibrils undergo a process of maturation. In this maturation process, the individual fibrils possibly go through some rearrangement leading to formation of thermodynamically favorable state(s), which manifests as heterogeneous and nonperiodic fibrils over time.

\section{METHODS}

Expression and Purification of $\alpha$ Syn. Wild-type and A30P, E46K, A53T, $\Delta 1-59$, and $\Delta 109-140$ mutants of $\alpha$ Syn were used in the present study. All the proteins were expressed and purified as described elsewhere. ${ }^{53}$

Fibrillization Assay and Thioflavin-T Fluorescence. Monomeric stocks of wt and disease mutant $\alpha$ Syn protein $(250 \mu \mathrm{L})$, stored at $-80{ }^{\circ} \mathrm{C}$, were thawed. Aggregation reactions for all the proteins were set up at $100 \mu \mathrm{M} \alpha$ Syn in $10 \mathrm{mM}$ Tris- $\mathrm{HCl}, 10 \mathrm{mM} \mathrm{NaCl}, 0.1$ $\mathrm{mM}$ EDTA at $\mathrm{pH}$ 7.4. All reactions were prepared in triplicate with volumes of $400 \mu \mathrm{L}$ each in $2 \mathrm{~mL}$ Lo-Bind round-bottom Eppendorf centrifuge tubes and were incubated at $37{ }^{\circ} \mathrm{C}$ with $500 \mathrm{rpm}$ orbital shaking in an Eppendorf Thermo-mixer comfort. Progress of fibrillization was followed by a Thioflavin- $\mathrm{T}$ (ThT) fluorescence assay as described elsewhere. ${ }^{53}$ Fibrils fibrillized at $4{ }^{\circ} \mathrm{C}$ were grown with same procedure with the Thermo-mixer placed in a cold room maintained at $4{ }^{\circ} \mathrm{C}$.

Atomic Force Microscopy: Fibril Morphology Analysis. AFM samples were prepared by adsorbing $10 \mu \mathrm{L}$ of 10 times diluted aggregation samples on freshly cleaved mica (Muscovite mica, V-1 quality, EMS) for 4 min, followed by 2 gentle washes with $100 \mu \mathrm{L}$ of fresh Milli-Q water and drying in a gentle stream of nitrogen gas. AFM images were acquired on a Bioscope Catalyst (Bruker, Santa Barbara, CA, USA) in soft tapping mode in air using a silicon probe, NSC36 tip $B$ with force constant of $1.75 \mathrm{~N} / \mathrm{m}$ (NanoAndMore $\mathrm{GmbH}$, Wetzlar, Germany). All images were captured with a resolution of 512 samples/ line with a scan rate of $0.5 \mathrm{~Hz}$. For each solution condition about 100 nonoverlapping fibrils were qualitatively analyzed by Scanning Probe Image Processor-6.02 (Image Metrology A/S, Hørsholm, Denmark) software; for quantitative analysis of height and periodicity, a custom written Matlab script using DIPimage toolbox (version 2.3, TU Delft, Delft, The Netherlands) was used. The script is based on quantitative analysis of AFM images as described elsewhere. ${ }^{40}$ To ensure reproducibility in the observed results, for all samples at a given time point, three independent aggregations were done in triplicate with a time delay of 1 to 3 months and about 30 images were acquired from one of the triplicates from each aggregation.

Dot Blot Analysis. A dot blot analysis was done to probe degradation of $\alpha$ Syn fibrils stored for 1 year. Aggregation reactions $(100 \mu \mathrm{L})$ were centrifuged at $21000 \mathrm{~g}$ for $30 \mathrm{~min}$ in an IEC Micromax microcentrifuge (Thermo Fisher Scientific Holding B.V., Breda, The Netherlands). Eighty microliters of the supernatant was discarded, and the pellet was resuspended in the rest of the $20 \mu \mathrm{L}$ buffer. ImmobilonP PVDF membrane (Millipore) was wetted in 100\% methanol for $15 \mathrm{~s}$ and rinsed in Mill-Q water. Five microliters of each of the samples was spotted on the wet membrane, and the membrane was soaked in $100 \%$ methanol for $10 \mathrm{~s}$. The blot was air-dried for $2 \mathrm{~h}$ followed by rewetting
(100\% methanol) of the membrane for $5 \mathrm{~s}$. The membrane was fixed in 0.4\% PFA (para-formaldehyde solution) in PBS at $\mathrm{pH} 7.4$ for 15 min at room temperature. Next, it was rinsed 3 times in PBS, pH 7.4. The blot was blocked in 5\% nonfat dry milk in TBS-T [Tris-buffered saline-Tween $(50 \mathrm{mM}$ Tris- $\mathrm{HCl}, 150 \mathrm{mM} \mathrm{NaCl}$ at $\mathrm{pH} 7.5$ and $0.2 \%$ $\mathrm{v} / \mathrm{v}$ Tween 20)] for $30 \mathrm{~min}$ and rinsed in TBS-T 3 times. Mouse IgG1 anti- $\alpha$ Syn 15-123 (610786, BD Biosciences) was diluted 1:2000 in 1\% nonfat dry milk in TBS-T and incubated overnight at $4{ }^{\circ} \mathrm{C}$. The blot was washed 3 times in TBS-T for 5 min each and incubated with antimouse HRP conjugated secondary antibody (G21234, Invitrogen), diluted 1:8000 in TBS-T, for $45 \mathrm{~min}$ at room temperature. The blot was washed 3 times in TBS, and the immunoreactive bands were detected using enhanced chemiluminescence (Super-Signal West, Pico-Thermo Scientific) followed by imaging on a Fluor Chem M imager (ProteinSimple, Westburg, Leusden, The Netherlands).

MALDI-TOF Mass Spectrometry. Samples for MALDI-TOF experiments were prepared by digesting plateau phase and matured fibrils of wt, A30P, E46K, A53T, and $\Delta 109-140$ fibrils with trypsin. Fibril samples, $140 \mu \mathrm{g}$, were centrifuged at $21000 \mathrm{~g}$ for $30 \mathrm{~min}$. The supernatant was removed, and the pellet was resuspended in $10 \mathrm{mM}$ $\left(\mathrm{NH}_{4}\right)_{2} \mathrm{CO}_{3}, \mathrm{pH} 7.4$, to a final volume of $100 \mu \mathrm{L}$. The samples were centrifuged again at $21000 \mathrm{~g}$ for $30 \mathrm{~min}$. After removal of the supernatant, the pellet was resuspended in $50 \mu \mathrm{L}$ of $10 \mathrm{mM}$ $\left(\mathrm{NH}_{4}\right)_{2} \mathrm{CO}_{3}, \mathrm{pH}$ 7.4. Next, the samples were digested with $400 \mathrm{ng}$ of sequencing grade modified trypsin (Promega) at $37{ }^{\circ} \mathrm{C}$ for $15 \mathrm{~h}$. The reaction was stopped by chilling on ice. One microliter of sample was deposited on a clean standard sample plate (M880675CD1) in triplicate with $1 \mu \mathrm{L}$ of universal matrix (TFA/acetonitrile $(50: 50 \mathrm{v} / \mathrm{v})$ ). The samples were allowed to air-dry. Mass spectra were acquired on a Waters MALDI SYNAPT high definition mass spectrometer equipped with a $355 \mathrm{~nm}$ third harmonic YAG laser in positive ion mode. The TOF linearity of the instrument was calibrated with PEG standard while bovine insulin was used as reference for the $\mathrm{m} / z$ calibration. To ensure reproducibility, mass spectra were measured 3 times using a different fibril sample for each measurement.

ATR-FTIR Experiment. Attenuated total reflectance Fourier transform infrared spectroscopy (ATR-FTIR) was done to investigate the structural features of plateau phase and 1 year old (matured) $\alpha$ Syn fibrils. Samples were prepared by centrifugation of $100 \mu \mathrm{L}$ of aggregation reactions at $21000 \mathrm{~g}$ for $30 \mathrm{~min}$ in an IEC Micromax microcentrifuge (Thermo Fisher Scientific Holding B.V., Breda, The Netherlands). The supernatant was discarded, and the pellet was resuspended in $20 \mu \mathrm{L}$ of buffer ( $10 \mathrm{mM}$ HEPES at $\mathrm{pH} 7.4$ ). The ATRFTIR spectra were acquired on a Bruker Equinox 55 spectrophotometer (Bruker Optics, Ettlingen, Germany) equipped with a Golden Gate reflectance accessory (Specac, Slough, United Kingdom). The internal reflection element was a diamond crystal $(2 \mathrm{~mm} \times 2 \mathrm{~mm})$ with an aperture angle of $45^{\circ}$ that yielded a single internal reflection. The crystal was cleaned with absolute ethanol, and $2-4 \mu \mathrm{L}$ of sample was applied to the diamond crystal. The sample was dried with a gentle flow of nitrogen gas. Excess salt was removed by placing $10 \mu \mathrm{L}$ of Milli-Q water on top of the sample and removing excess water with a micropipette. The sample was dried again with a gentle flow of nitrogen gas. One hundred twenty-eight coadded spectra were collected for each sample with a resolution of $2 \mathrm{~cm}^{-1}$. For data analysis, baseline correction was done by a straight line passing through ordinates $1753,1712,1593,1482$, and $1425 \mathrm{~cm}^{-1}$, followed by water vapor subtraction. Then Fourier self-deconvolution was applied using a Lorentzian and Gaussian line shape for deconvolution and apodization, respectively. The identified peaks were curve fitted on nondeconvoluted spectra. Area under each deconvoluted peak was quantified to estimate the secondary structure of the sample. ${ }^{67}$

RMC and Reversibility Analysis. Determination of the fraction of monomers left in the aggregation reaction was done by centrifugation of $100 \mu \mathrm{L}$ aliquots of aggregation reactions at $21000 \mathrm{~g}$ for $1 \mathrm{~h}$ in an IEC Micromax microcentrifuge (Thermo Fisher Scientific Holding B.V., Breda, The Netherlands). Eighty microliters of supernatant was aspirated and analyzed for residual monomer concentration. Absorbance at 280 and $330 \mathrm{~nm}$ was measured from $2 \mu \mathrm{L}$ samples on a NanoDrop ND-1000 spectrophotometer (Isogen Life Science 
B.V., The Netherlands). The absorption values at $280 \mathrm{~nm}$ were corrected for scattering contributions before calculating the residual monomer concentration. ${ }^{68}$ For reversibility analysis, $100 \mu \mathrm{L}$ of the plateau phase reaction was centrifuged at $21000 \mathrm{~g}$ for $1 \mathrm{~h}, 80 \mu \mathrm{L}$ of the supernatant was removed, and RMC was determined as mentioned above. The fibril pellet was resuspended in $80 \mu \mathrm{L}$ of fresh buffer (total volume $100 \mu \mathrm{L}$ ) of $10 \mathrm{mM}$ Tris- $\mathrm{HCl}, 10 \mathrm{mM} \mathrm{NaCl}$, and $0.1 \mathrm{mM}$ EDTA at $\mathrm{pH} 7.4$ and stored at $37^{\circ} \mathrm{C}$ for 1 week. The procedure was repeated 3 times each while storing the samples at 37 and $4{ }^{\circ} \mathrm{C}$. At every alternate week $5 \mu \mathrm{L}$ of the fibril sample was analyzed by ThT fluorescence assay for detection of $\beta$-sheet positive fibrils.

\section{ASSOCIATED CONTENT}

\section{S Supporting Information}

The Supporting Information is available free of charge on the ACS Publications website at DOI: 10.1021/acschemneuro.6b00287.

Comparison of $\alpha$ Syn aggregation kinetics, representative AFM images of fibrils from disease mutants over 6 months, from wt and mutants at 1 year, and from wt fibrils fibrillized at $4{ }^{\circ} \mathrm{C}$ after 3 months and 1 year, summaries of mean fibril periodicity, fraction of periodic fibrils, and mean fibril height, scatter plots of heights and periodicities of fibrils, example of fibril height determination, dot blot using anti- $\alpha$ Syn on plateau phase and matured fibrils, lists of trypsin cleavage sites and possible peptide masses in $\alpha$ Syn, summaries of average molecular masses of C-terminal peptides from plateau phase and matured fibrils, mass spectra of trypsin digested fibrils, and curve fitting of amide I region of ATR-FTIR spectra (PDF)

\section{AUTHOR INFORMATION}

\section{Corresponding Author}

*E-mail: v.subramaniam@vu.nl.

ORCID ${ }^{\circ}$

Arshdeep Sidhu: 0000-0002-2851-1019

\section{Present Address}

${ }^{\#}$ A.S.: Department of Molecular Genetics, Erasmus MC, P.O. Box 2040, 3000 CA Rotterdam, The Netherlands.

\section{Author Contributions}

A.S., I.S.-N., V.R., and V.S. designed the research; A.S. and V.R. performed the research; A.S., I.S.-N., V.R., M.M.A.E.C., and V.S. analyzed data; A.S., I.S.-N., V.R., M.M.A.E.C., and V.S. wrote the paper.

\section{Funding}

This work is supported by NanoNextNL, a micro- and nanotechnology consortium of the Government of The Netherlands and 130 partners. We also acknowledge support from the Foundation for Fundamental Research on Matter (FOM), which is part of Netherlands Organization for Scientific Research (NWO). V.R. is Senior Research Associate for the F.R.S.-FNRS (Belgium) and acknowledges support from the F.R.S.-FNRS (PDR No. 70214.12) and the SAO-FRA (Grant No. 14025).

\section{Notes}

The authors declare no competing financial interest.

\section{ACKNOWLEDGMENTS}

The authors thank Kirsten van Leijenhorst-Groener, Yvonne Kraan, and Nathalie Schilderink for protein expression and purification and Dr. Martin Bennink, Kees van der Werf, and
Robert Molenaar for advice on AFM. We also thank Henk van Wolferen for mass spectrometry measurements at the BioNanoLab of MESA+ Institute for Nanotechnology, University of Twente.

\section{ABBREVIATIONS}

$\alpha$ Syn, $\alpha$-synuclein; AFM, atomic force microscopy; wt, wildtype; A30P, $\alpha$ Syn protein sequence with residue 30 (alanine) mutated to proline; E46K, $\alpha$ Syn protein sequence with residue 46 (glutamate) mutated to lysine; A53T, $\alpha$ Syn protein sequence with residue 53 (alanine) mutated to threonine; PD, Parkinson's disease; ThT, Thioflavin-T; RMC, residual monomer concentration; CMC, critical monomer concentration; MALDI-TOF, matrix assisted laser desorption ionization-time-of-flight; ATR-FTIR, attenuated total reflectance-Fourier transform infrared spectroscopy; HEPES, 4-(2hydroxyethyl)-1-piperazineethanesulfonic acid

\section{REFERENCES}

(1) Westermark, G. T., and Westermark, P. (2013) Islet amyloid polypeptide and diabetes. Curr. Protein Pept. Sci. 14, 330-337.

(2) Breydo, L., Wu, J. W., and Uversky, V. N. (2012) Alpha-synuclein misfolding and Parkinson's disease. Biochim. Biophys. Acta, Mol. Basis Dis. 1822, 261-285.

(3) Chiti, F., and Dobson, C. M. (2006) Protein misfolding, functional amyloid, and human disease. Annu. Rev. Biochem. 75, 333366.

(4) Harper, J. D., Wong, S. S., Lieber, C. M., and Lansbury, P. T., Jr. (1999) Assembly of A beta amyloid protofibrils: an in vitro model for a possible early event in Alzheimer's disease. Biochemistry 38, 89728980.

(5) Uversky, V. N., Li, J., and Fink, A. L. (2001) Metal-triggered structural transformations, aggregation, and fibrillation of human alpha-synuclein. A possible molecular NK between Parkinson's disease and heavy metal exposure. J. Biol. Chem. 276, 44284-44296.

(6) Jimenez, J. L., Nettleton, E. J., Bouchard, M., Robinson, C. V., Dobson, C. M., and Saibil, H. R. (2002) The protofilament structure of insulin amyloid fibrils. Proc. Natl. Acad. Sci. U. S. A. 99, 9196-9201.

(7) Khurana, R., Ionescu-Zanetti, C., Pope, M., Li, J., Nielson, L., Ramirez-Alvarado, M., Regan, L., Fink, A. L., and Carter, S. A. (2003) A general model for amyloid fibril assembly based on morphological studies using atomic force microscopy. Biophys. J. 85, 1135-1144.

(8) Pronchik, J., He, X., Giurleo, J. T., and Talaga, D. S. (2010) In vitro formation of amyloid from alpha-synuclein is dominated by reactions at hydrophobic interfaces. J. Am. Chem. Soc. 132, 9797-9803.

(9) Hoyer, W., Antony, T., Cherny, D., Heim, G., Jovin, T. M., and Subramaniam, V. (2002) Dependence of alpha-synuclein aggregate morphology on solution conditions. J. Mol. Biol. 322, 383-393.

(10) Raman, B., Chatani, E., Kihara, M., Ban, T., Sakai, M., Hasegawa, K., Naiki, H., Rao, Ch. M., and Goto, Y. (2005) Critical balance of electrostatic and hydrophobic interactions is required for beta 2microglobulin amyloid fibril growth and stability. Biochemistry 44, 1288-1299.

(11) Nielsen, L., Khurana, R., Coats, A., Frokjaer, S., Brange, J., Vyas, S., Uversky, V. N., and Fink, A. L. (2001) Effect of environmental factors on the kinetics of insulin fibril formation: elucidation of the molecular mechanism. Biochemistry 40, 6036-6046.

(12) Morel, B., Varela, L., Azuaga, A. I., and Conejero-Lara, F. (2010) Environmental conditions affect the kinetics of nucleation of amyloid fibrils and determine their morphology. Biophys. J. 99, 3801-3810.

(13) Guilliams, T., El-Turk, F., Buell, A. K., O’Day, E. M., Aprile, F. A., Esbjorner, E. K., Vendruscolo, M., Cremades, N., Pardon, E., Wyns, L., Welland, M. E., Steyaert, J., Christodoulou, J., Dobson, C. M., and De Genst, E. (2013) Nanobodies raised against monomeric alphasynuclein distinguish between fibrils at different maturation stages. $J$. Mol. Biol. 425, 2397-2411. 
(14) Nystrom, S., Psonka-Antonczyk, K. M., Ellingsen, P. G., Johansson, L. B., Reitan, N., Handrick, S., Prokop, S., Heppner, F. L., Wegenast-Braun, B. M., Jucker, M., Lindgren, M., Stokke, B. T., Hammarstrom, P., and Nilsson, K. P. (2013) Evidence for agedependent in vivo conformational rearrangement within Abeta amyloid deposits. ACS Chem. Biol. 8, 1128-1133.

(15) Ma, J., Komatsu, H., Kim, Y. S., Liu, L., Hochstrasser, R. M., and Axelsen, P. H. (2013) Intrinsic structural heterogeneity and long-term maturation of amyloid beta peptide fibrils. ACS Chem. Neurosci. 4, 1236-1243.

(16) Vilar, M., Chou, H. T., Luhrs, T., Maji, S. K., Riek-Loher, D., Verel, R., Manning, G., Stahlberg, H., and Riek, R. (2008) The fold of alpha-synuclein fibrils. Proc. Natl. Acad. Sci. U. S. A. 105, 8637-8642.

(17) Heise, H., Hoyer, W., Becker, S., Andronesi, O. C., Riedel, D., and Baldus, M. (2005) Molecular-level secondary structure, polymorphism, and dynamics of full-length alpha-synuclein fibrils studied by solid-state NMR. Proc. Natl. Acad. Sci. U. S. A. 102, 15871-15876.

(18) Fandrich, M., Meinhardt, J., and Grigorieff, N. (2009) Structural polymorphism of Alzheimer Abeta and other amyloid fibrils. Prion 3, 89-93.

(19) Kodali, R, and Wetzel, R. (2007) Polymorphism in the intermediates and products of amyloid assembly. Curr. Opin. Struct. Biol. 17, 48-57.

(20) Pellarin, R., Schuetz, P., Guarnera, E., and Caflisch, A. (2010) Amyloid fibril polymorphism is under kinetic control. J. Am. Chem. Soc. 132, 14960-14970.

(21) Petkova, A. T., Leapman, R. D., Guo, Z., Yau, W. M., Mattson, M. P., and Tycko, R. (2005) Self-propagating, molecular-level polymorphism in Alzheimer's beta-amyloid fibrils. Science 307, 262265.

(22) Bousset, L., Pieri, L., Ruiz-Arlandis, G., Gath, J., Jensen, P. H., Habenstein, B., Madiona, K., Olieric, V., Bockmann, A., Meier, B. H., and Melki, R. (2013) Structural and functional characterization of two alpha-synuclein strains. Nat. Commun. 4, 2575.

(23) Paravastu, A. K., Leapman, R. D., Yau, W. M., and Tycko, R. (2008) Molecular structural basis for polymorphism in Alzheimer's beta-amyloid fibrils. Proc. Natl. Acad. Sci. U. S. A. 105, 18349-18354. (24) Gath, J., Bousset, L., Habenstein, B., Melki, R., Bockmann, A., and Meier, B. H. (2014) Unlike Twins: An NMR Comparison of Two alpha-Synuclein Polymorphs Featuring Different Toxicity. PLoS One 9, e90659.

(25) Onuchic, J. N., Luthey-Schulten, Z., and Wolynes, P. G. (1997) Theory of protein folding: the energy landscape perspective. Annu. Rev. Phys. Chem. 48, 545-600.

(26) Dill, K. A., and Chan, H. S. (1997) From Levinthal to pathways to funnels. Nat. Struct. Biol. 4, 10-19.

(27) Dobson, C. M. (2003) Protein folding and misfolding. Nature 426, 884-890.

(28) Eaton, W. A., Thompson, P. A., Chan, C. K., Hage, S. J., and Hofrichter, J. (1996) Fast events in protein folding. Structure 4, 11331139.

(29) Guo, Z. Y., and Thirumalai, D. (1995) Kinetics of ProteinFolding - Nucleation Mechanism, Time Scales, and Pathways. Biopolymers 36, 83-102.

(30) Fink, A. L. (2006) The aggregation and fibrillation of alphasynuclein. Acc. Chem. Res. 39, 628-634.

(31) Buell, A. K., Galvagnion, C., Gaspar, R., Sparr, E., Vendruscolo, M., Knowles, T. P., Linse, S., and Dobson, C. M. (2014) Solution conditions determine the relative importance of nucleation and growth processes in alpha-synuclein aggregation. Proc. Natl. Acad. Sci. U. S. A. 111, 7671-7676.

(32) Chiti, F., Taddei, N., Baroni, F., Capanni, C., Stefani, M., Ramponi, G., and Dobson, C. M. (2002) Kinetic partitioning of protein folding and aggregation. Nat. Struct. Biol. 9, 137-143.

(33) Sidhu, A., Segers-Nolten, I., and Subramaniam, V. (2016) Conformational Compatibility Is Essential for Heterologous Aggregation of alpha-Synuclein. ACS Chem. Neurosci. 7, 719-727.
(34) Conway, K. A., Harper, J. D., and Lansbury, P. T. (1998) Accelerated in vitro fibril formation by a mutant alpha-synuclein linked to early-onset Parkinson disease. Nat. Med. 4, 1318-1320.

(35) Narhi, L., Wood, S. J., Steavenson, S., Jiang, Y., Wu, G. M., Anafi, D., Kaufman, S. A., Martin, F., Sitney, K., Denis, P., Louis, J. C., Wypych, J., Biere, A. L., and Citron, M. (1999) Both familial Parkinson's disease mutations accelerate alpha-synuclein aggregation. J. Biol. Chem. 274, 9843-9846.

(36) Giasson, B. I., Uryu, K., Trojanowski, J. Q., and Lee, V. M. (1999) Mutant and wild type human alpha-synucleins assemble into elongated filaments with distinct morphologies in vitro. J. Biol. Chem. 274, 7619-7622.

(37) Li, J., Uversky, V. N., and Fink, A. L. (2001) Effect of familial Parkinson's disease point mutations A30P and A53T on the structural properties, aggregation, and fibrillation of human alpha-synuclein. Biochemistry 40, 11604-11613.

(38) Choi, W., Zibaee, S., Jakes, R., Serpell, L. C., Davletov, B., Crowther, R. A., and Goedert, M. (2004) Mutation E46K increases phospholipid binding and assembly into filaments of human alphasynuclein. FEBS Lett. 576, 363-368.

(39) Greenbaum, E. A., Graves, C. L., Mishizen-Eberz, A. J., Lupoli, M. A., Lynch, D. R., Englander, S. W., Axelsen, P. H., and Giasson, B. I. (2005) The E46K mutation in alpha-synuclein increases amyloid fibril formation. J. Biol. Chem. 280, 7800-7807.

(40) van Raaij, M. E., Segers-Nolten, I. M., and Subramaniam, V. (2006) Quantitative morphological analysis reveals ultrastructural diversity of amyloid fibrils from alpha-synuclein mutants. Biophys. J. 91, L96-98.

(41) Ghosh, D., Mondal, M., Mohite, G. M., Singh, P. K., Ranjan, P., Anoop, A., Ghosh, S., Jha, N. N., Kumar, A., and Maji, S. K. (2013) The Parkinson's disease-associated H50Q mutation accelerates alphaSynuclein aggregation in vitro. Biochemistry 52, 6925-6927.

(42) Ghosh, D., Sahay, S., Ranjan, P., Salot, S., Mohite, G. M., Singh, P. K., Dwivedi, S., Carvalho, E., Banerjee, R., Kumar, A., and Maji, S. K. (2014) The newly discovered Parkinson's disease associated Finnish mutation (A53E) attenuates alpha-synuclein aggregation and membrane binding. Biochemistry 53, 6419-6421.

(43) Peelaerts, W., Bousset, L., Van der Perren, A., Moskalyuk, A., Pulizzi, R., Giugliano, M., Van den Haute, C., Melki, R., and Baekelandt, V. (2015) alpha-Synuclein strains cause distinct synucleinopathies after local and systemic administration. Nature $522,340$.

(44) Goedert, M., Falcon, B., Clavaguera, F., and Tolnay, M. (2014) Prion-like mechanisms in the pathogenesis of tauopathies and synucleinopathies. Curr. Neurol. Neurosci. Rep. 14, 495.

(45) Dunning, C. J., George, S., and Brundin, P. (2013) What's to like about the prion-like hypothesis for the spreading of aggregated alpha-synuclein in Parkinson disease? Prion 7, 92-97.

(46) George, S., Rey, N. L., Reichenbach, N., Steiner, J. A., and Brundin, P. (2013) alpha-Synuclein: the long distance runner. Brain Pathol. 23, 350-357.

(47) Guo, J. L., Covell, D. J., Daniels, J. P., Iba, M., Stieber, A., Zhang, B., Riddle, D. M., Kwong, L. K., Xu, Y., Trojanowski, J. Q., and Lee, V. M. (2013) Distinct alpha-synuclein strains differentially promote tau inclusions in neurons. Cell 154, 103-117.

(48) Watts, J. C., Giles, K., Oehler, A., Middleton, L., Dexter, D. T., Gentleman, S. M., DeArmond, S. J., and Prusiner, S. B. (2013) Transmission of multiple system atrophy prions to transgenic mice. Proc. Natl. Acad. Sci. U. S. A. 110, 19555-19560.

(49) Prusiner, S. B., Woerman, A. L., Mordes, D. A., Watts, J. C., Rampersaud, R., Berry, D. B., Patel, S., Oehler, A., Lowe, J. K., Kravitz, S. N., Geschwind, D. H., Glidden, D. V., Halliday, G. M., Middleton, L. T., Gentleman, S. M., Grinberg, L. T., and Giles, K. (2015) Evidence for $\alpha$-synuclein prions causing multiple system atrophy in humans with parkinsonism. Proc. Natl. Acad. Sci. U. S. A. 112, E5308-E5317.

(50) Tanaka, M., Collins, S. R., Toyama, B. H., and Weissman, J. S. (2006) The physical basis of how prion conformations determine strain phenotypes. Nature 442, 585-589. 
(51) Shikiya, R. A., Ayers, J. I., Schutt, C. R., Kincaid, A. E., and Bartz, J. C. (2010) Coinfecting prion strains compete for a limiting cellular resource. J. Virol. 84, 5706-5714.

(52) Dickinson, A. G., Fraser, H., and Outram, G. W. (1975) Scrapie incubation time can exceed natural lifespan. Nature 256, 732-733.

(53) Sidhu, A., Segers-Nolten, I., and Subramaniam, V. (2014) Solution conditions define morphological homogeneity of alphasynuclein fibrils. Biochim. Biophys. Acta, Proteins Proteomics 1844, 2127-2134.

(54) Semerdzhiev, S. A., Dekker, D. R., Subramaniam, V., and Claessens, M. M. (2014) Self-assembly of protein fibrils into suprafibrillar aggregates: bridging the nano- and mesoscale. ACS Nano 8, 5543-5551.

(55) Campioni, S., Carret, G., Jordens, S., Nicoud, L., Mezzenga, R., and Riek, R. (2014) The Presence of an Air-Water Interface Affects Formation and Elongation of alpha-Synuclein Fibrils. J. Am. Chem. Soc. 136, 2866-2875.

(56) Gershenson, A., Gierasch, L. M., Pastore, A., and Radford, S. E. (2014) Energy landscapes of functional proteins are inherently risky. Nat. Chem. Biol. 10, 884-891.

(57) Arosio, P., Knowles, T. P., and Linse, S. (2015) On the lag phase in amyloid fibril formation. Phys. Chem. Chem. Phys. 17, 7606-7618.

(58) Lemkau, L. R., Comellas, G., Kloepper, K. D., Woods, W. S., George, J. M., and Rienstra, C. M. (2012) Mutant protein A30P alphasynuclein adopts wild-type fibril structure, despite slower fibrillation kinetics. J. Biol. Chem. 287, 11526-11532.

(59) Heise, H., Celej, M. S., Becker, S., Riedel, D., Pelah, A., Kumar, A., Jovin, T. M., and Baldus, M. (2008) Solid-state NMR reveals structural differences between fibrils of wild-type and disease-related A53T mutant alpha-synuclein. J. Mol. Biol. 380, 444-450.

(60) Nielsen, S. B., Macchi, F., Raccosta, S., Langkilde, A. E., Giehm, L., Kyrsting, A., Svane, A. S., Manno, M., Christiansen, G., Nielsen, N. C., Oddershede, L., Vestergaard, B., and Otzen, D. E. (2013) Wildtype and A30P mutant alpha-synuclein form different fibril structures. PLoS One 8, e67713.

(61) Comellas, G., Lemkau, L. R., Nieuwkoop, A. J., Kloepper, K. D., Ladror, D. T., Ebisu, R., Woods, W. S., Lipton, A. S., George, J. M., and Rienstra, C. M. (2011) Structured regions of alpha-synuclein fibrils include the early-onset Parkinson's disease mutation sites. J. Mol. Biol. $411,881-895$.

(62) Sahay, S., Ghosh, D., Dwivedi, S., Anoop, A., Mohite, G. M., Kombrabail, M., Krishnamoorthy, G., and Maji, S. K. (2015) Familial Parkinson Disease-associated Mutations Alter the Site-specific Microenvironment and Dynamics of alpha-Synuclein. J. Biol. Chem. 290, 7804-7822.

(63) Chen, M., Margittai, M., Chen, J., and Langen, R. (2007) Investigation of alpha-synuclein fibril structure by site-directed spin labeling. J. Biol. Chem. 282, 24970-24979.

(64) Sweers, K. K., van der Werf, K. O., Bennink, M. L., and Subramaniam, V. (2012) Atomic force microscopy under controlled conditions reveals structure of $\mathrm{C}$-terminal region of alpha-synuclein in amyloid fibrils. ACS Nano 6, 5952-5960.

(65) Ikenoue, T., Lee, Y. H., Kardos, J., Saiki, M., Yagi, H., Kawata, Y., and Goto, Y. (2014) Cold Denaturation of alpha-Synuclein Amyloid Fibrils. Angew. Chem., Int. Ed. 53, 7799-7804.

(66) Jucker, M., and Walker, L. C. (2013) Self-propagation of pathogenic protein aggregates in neurodegenerative diseases. Nature $501,45-51$.

(67) Goormaghtigh, E., Raussens, V., and Ruysschaert, J. M. (1999) Attenuated total reflection infrared spectroscopy of proteins and lipids in biological membranes. Biochim. Biophys. Acta, Rev. Biomembr. 1422, $105-185$.

(68) Grimsley, G. R., and Pace, C. N. (2003) Spectrophotometric Determination of Protein Concentration. Curr. Protoc. Protein Sci., 3.1.1-3.1.9. 